\title{
Epidemiology of mental health problems in adults with learning disability: an update
}

\author{
Elita Smiley
}

\begin{abstract}
Epidemiological studies measuring prevalence rates and factors associated with mental health problems in adults with learning disability have produced very different and sometimes contradictory results, mainly because of methodological problems. Consequently, much of the epidemiology of mental health problems in this population is still unknown, although improved methodology and additional information are emerging. This article describes the methodological difficulties of studies, the reported prevalence rates in the context of these difficulties and what is currently known about the factors associated with mental health problems in this population.
\end{abstract}

As recently as the 1980s, it was still generally believed that people with learning disability did not have the cognitive capacity to experience mental health problems and that behavioural disturbances were attributable to their learning disability. Over the past 25 years there has been considerable interest and effort in advancing our knowledge and understanding of mental health problems in learning-disabled adults and it is now accepted that they do experience the same mental ill-health as adults without such disability and that they are probably more vulnerable. However, to date, epidemiological studies measuring prevalence rates and factors associated with mental health problems in this population have produced very different and sometimes contradictory results, mainly because of methodological difficulties such as those summarised in Box 1.

\section{Methodological challenges}

An accurate measurement of mental health problems in people with learning disabilities requires a valid

\section{Box 1 Key methodological challenges}

- The definition of learning disability

- The definition of mental health problems

- The representativeness of study samples

- The method of identification and assessment of cases

- The use of appropriate diagnostic criteria and reliable measurement of both the learning disability and the mental health problems. Both are complicated and the methods used need to be taken into consideration when evaluating the results of epidemiological studies.

\section{Definition of learning disability and identification of populations}

The effect that the definition of learning disability can have on the results of epidemiological studies is illustrated by the example of the change in the American Association on Mental Retardation's definition of mental retardation. In 1973 this was changed from one standard deviation below the mean on an IQ test to two standard deviations below, with the result that many individuals included in learning disability studies before 1973 would now be excluded.

Identifying populations of adults with learning disability from case registers or those in receipt of specifically targeted social funding or learning disability services has a good ascertainment rate for moderate-to-profound learning disability but is not so good for mild learning disability. Typically, adults with mild learning disability are not known to learning disability services or, if they are, it is because they have additional problems such as mental illness, and this leads to a biased sample. Furthermore, there is considerable variation in the methods used to set up and maintain such case registers and therefore samples taken from different case registers, even within the UK, are not always directly comparable.

Elita Smiley is a specialist registrar in learning disability and general adult psychiatry on the West of Scotland Higher Training Scheme and an honorary clinical teacher at the University of Glasgow (Department of Learning Disability Psychiatry, West House, Gartnavel Royal Hospital, 1055 Great Western Road, Glasgow G12 0XH, UK. Tel: 0141211 3878; e-mail: ElitaSmiley@tinyonline.co.uk). Her main research interest is the epidemiology of mental health in adults with learning disability. She is currently involved in a large population-based study measuring the prevalence and incidence of mental ill-health in adults with learning disability in Glasgow. 
Accurate epidemiological information can be obtained only by using reliable and valid measurements on population-based samples. The ideal method would be to screen everyone in a certain area for learning disability and then to further screen those identified for mental health problems using clinical assessment and appropriate diagnostic criteria, as discussed below. However, this would be costly and time consuming. A few studies have used population-based samples, but they are limited by small sample sizes.

\section{Definition and identification of mental health problems}

In prevalence studies, many researchers have used terms such as mental illness, mental disorder, psychiatric illness, psychiatric disorder, emotional problems and behavioural disorder without detailed definition. Some have excluded personality disorder and behavioural disorder from their reported prevalence rates, whereas others have included them. This difference in the types of disorder counted can have a considerable effect on reported prevalence rates and makes the comparison of studies complicated.

\section{Diagnostic criteria}

The diagnostic criteria used have also varied. Some studies have used the World Health Organization's International Classification of Diseases (ICD) or the American Psychiatric Association's Diagnostic and Statistical Manual of Mental Disorders (DSM), but these systems were designed for use in the general population and are not entirely appropriate for people with learning disability, mainly because of their reliance on the subjective report of symptomatology. In response to this, individual researchers have modified these criteria to make them more appropriate for people with learning disability, but frequently these modifications have not been reported, making the interpretation and comparison of studies unreliable.

The Diagnostic Criteria for Adults with Learning Disability (DC-LD) published by the Royal College of Psychiatrists in 2001 attempt to address this issue. DC-LD is a diagnostic system developed in recognition of the limitations of using ICD-10 (World Health Organization, 1992) and DSM-IV (American Psychiatric Association, 1994) in adults with learning disability. The criteria represent a consensus of professional opinion in the UK and Ireland and have very good face validity, but they are yet to be fully evaluated with regard to their psychometric properties. None the less, DC-LD is a much welcomed addition which should prove useful in epidemiological studies by allowing researchers to produce comparable results.

\section{Assessment tools}

The identification and correct diagnosis of mental health problems in learning-disabled adults is complex and can be highly challenging even for the most experienced clinicians. People with learning disability often do not recognise their own symptoms or cannot report them and they have to rely on others for accessing their healthcare. Their carers do not reliably recognise symptomatology and often do not realise its significance because of a lack of understanding and knowledge of mental health problems in learning disability.

Several tools have been developed to assist in the identification of mental health problems in people with learning disability. These include the Aberrant Behavior Checklist (Aman et al, 1985), the Psychopathology Instrument for Mentally Retarded Adults (Matson et al, 1984), the Emotional Disorder Rating Scale for Developmental Disabilities (Feinstein et al, 1988) and the Reiss Screen for Maladaptive Behavior (Reiss, 1988). Each of these has been used in studies but, as they are all screening rather than diagnostic instruments and little work has been done on their psychometric properties, the validity of the results obtained is limited.

\section{The PAS-ADD}

The more recent Psychiatric Assessment Schedule for Adults with a Developmental Disability (PASADD; Moss et al, 1993b), developed by the Hester Adrian Research Centre, has been shown to have both reasonable reliability and validity (Costello et al, 1997; Moss et al, 1997, 1998; Prosser et al, 1998). This makes it one of the most useful tools currently available for the identification and diagnosis of psychiatric disorder in adults with learning disability. The PAS-ADD is a semi-structured interview (derived from the Schedule for Clinical Assessment in Neuropsychiatry (SCAN) and version 10 of the Psychiatric Assessment Schedule) designed to produce a diagnosis according to ICD-10. The instrument is also available in two other forms. The Mini PAS-ADD provides a framework for professionals to collect relevant information on psychiatric symptomatology without the need for interviewing and is aimed at case identification rather than diagnosis. The PAS-ADD Checklist is a questionnaire for carers and staff to help them decide whether an individual requires further assessment, and it is a useful screening tool for identifying possible cases of mental ill-health.

\section{Confounding factors}

The method used for assessing psychopathology can also have a significant effect on the prevalence rate found. This is demonstrated in a study by Reiss 
(1990) in which the prevalence rate of mental health problems for the same sample was $11.7 \%$ when diagnoses were taken from the case notes, 39\% using the Reiss Screen for Maladaptive Behavior and 59\% after clinical assessment.

Another methodological issue encountered in studying mental health problems in adults with learning disability is that both conditions (mental illness and learning disability) can confound the detection of either. Many people with serious mental health problems perform poorly in IQ tests and may be mistakenly classified as having a learning disability. The opposite may also happen, with the low functioning of a person with mental illness being ascribed to the effect of the illness, thus missing the underlying learning disability. Furthermore, there is the issue of diagnostic overshadowing, with mental health symptomatology being mistakenly assumed to be due to the learning disability rather than to a mental illness. This highlights the importance of eliciting and correctly interpreting psychopathology, even when assessment tools are used.

\section{Sampling bias}

Epidemiological studies are seriously affected by the target population chosen for study and this has been particularly problematic in learning disability. Many prevalence studies have used institutionalised or psychiatric out-patient populations, both of which are likely to have a high prevalence of mental health problems. However, a significant number of learningdisabled people with mental health problems are unknown to services, and using samples that are not population-based excludes this group.

\section{Some reported prevalence rates}

In a sample of 402 people over 14 years of age, taken from a register of individuals in contact with learning disability services (which included people receiving hospital day care and supervised residential care) in the London borough of Camberwell, Corbett (1979) found a total prevalence of ICD-8 mental health problems of $46 \%$. This rate includes problem behaviour and past psychiatric disorder but not dementia. The study used an initial screen of behavioural disturbance or a history of psychiatric disorder in case notes to identify individuals who then underwent a psychiatric assessment.

Lund (1985b) identified a random sample of 302 people aged over 19 years from the Danish National Service for the Mentally Retarded register and used items in the Handicaps, Behaviour and Skills Schedule plus a routine psychiatric checklist in his examination of each person. He coded the results using modified Feighner and DSM-III criteria and found a point prevalence of $28 \%$. This rate includes problem behaviour but not past psychiatric disorder.

Cooper (1997) identified a random sample of 81 individuals aged 20-64 years and everyone over 64 years of age from the Leicestershire Learning Disabilities Register (total sample size of 207). Each participant underwent clinical assessment using a variety of tools, and diagnoses were classified according to ICD-10 Diagnostic Criteria for Research with modifications by the author. She found a lifetime prevalence rate for all psychiatric disorders, including possible dementia, Rett syndrome and problem behaviour, of $49.2 \%$.

Deb et al (2001a) identified a random sample of 101 adults aged 16-64 years from a local social service case register in Wales. Of these, 90 were screened using the Mini PAS-ADD and those that were identified as possible cases then underwent the full PAS-ADD interview (11 participants with severe learning disability were excluded from the sample because of the questionable reliability of using the PAS-ADD interview in this population). Diagnoses were classified according to ICD-10 and a point prevalence rate of $14.4 \%$ was reported. This rate excludes problem behaviour, dementia, autism, alcohol problems, and schizophrenia and bipolar affective disorder not in episode and is for mild and moderate levels of learning disability only.

Although the prevalence rates in the above studies vary considerably (mainly owing to different definitions of mental health, diagnostic criteria and assessment methods) all the reported rates except for that of Deb et al (2001a) are much higher than the rate for the general population. Bearing in mind the limitations of these studies, it is likely that the point prevalence of mental health problems (including problem behaviour) in adults with learning disability lies between 30 and 50\%. The rate reported by Deb et al is much lower primarily because of the use of unmodified ICD-10 criteria, the exclusion of problem behaviour and autism (which are particularly prevalent in learning disability) and the exclusion of participants with severe learning disability.

\section{Total prevalence and level of ability}

Studies examining the prevalence of mental health problems across differing levels of ability have produced conflicting results. Some have found higher rates of psychiatric disorder in people with mild learning disability than in those with severe or profound disability, whereas others have found lower rates. The higher rates in mild learning disability are at least partly explained by biased sampling. This has two causes. First, those with mild learning disability that are known to learning disability services tend to have additional problems 
such as psychiatric, physical or social disorders. Second, it is difficult to identify and diagnose a psychiatric disorder according to standard diagnostic criteria in people with severe and profound learning disability.

When considering possible aetiological factors such as epilepsy, physical disability and sensory impairment, it makes sense that there would be an increased rate of mental health problems in people with more severe learning disability and this has been described by Cooper \& Bailey (2001). However, before this issue can be answered definitively further work needs to be done on population-based samples large enough to demonstrate differences and using assessment methods and diagnostic criteria appropriate for all levels of ability. Also, in view of the different manifestations of different disorders across different levels of ability, examining specific disorders rather than total prevalence rates may be more relevant in studying this question.

\section{Total prevalence and age}

As with the level of ability, the literature has inconsistent findings with regard to total prevalence and age. Several studies have found no differences in the age distributions of groups with and without mental health problems, some have found a higher prevalence of mental health problems in older adults and some have found a reduced prevalence in older age groups (Day, 1985). Cooper (1997) found the prevalence of psychiatric morbidity to be $68.7 \%$ in a group aged over 64 years and $47.9 \%$ in a group aged 20-64 years. Most of this additional morbidity was accounted for by increased rates of depression, anxiety disorders and dementia. Deb et al (2001a) reported a statistically significant association between the rate of psychiatric illness and increasing age in his study of 101 learning-disabled adults aged 16-64 years. Factors likely to increase the prevalence of mental health problems in older people include the increasing sensory deficits and physical health problems associated with age, the cumulative effect of life events and the association of certain conditions such as dementia with age. However, the effect of differential mortality, i.e. those with more severe learning disability die at a younger age than those with milder levels because of associated physical disabilities, probably operates in the opposite direction. Again, larger studies with better methodology are required to determine whether any specific age group is more at risk than others.

\section{Total prevalence and gender}

The relationship between gender and mental health problems in adults with learning disability is also still unclear. Most studies have found that gender is unrelated to the overall rate of psychiatric disorder. However, since in the general population female gender is associated with a higher rate of affective and anxiety disorder and male gender with a higher rate of psychosis, it might be more helpful to look at the effect of gender on specific disorders rather than total prevalence rates. Studies so far in adults with learning disability have been of numbers insufficient to demonstrate such an effect, if it is there.

\section{Total prevalence and epilepsy}

In the general population it is widely accepted that epilepsy confers an increased risk for mental health disturbances. Depression, anxiety disorders and psychosis are all common in people with epilepsy. However, it is uncertain whether this is also the case in people with learning disability. Lund (1985a) found a rate of $52 \%$ for psychiatric diagnosis in people who had had seizures within the previous year compared with $26 \%$ in those without seizures, and Corbett (1979) found a rate of $60.8 \%$ in those with epilepsy and $40.4 \%$ in this without. Deb \& Joyce (1998), however, found no increased rate of problem behaviour or psychiatric illness in learning-disabled people with epilepsy. More recently, a study by Espie et al (2003) found a rate of psychiatric disorder according to the PAS-ADD Checklist of $33 \%$ in a sample of 186 people with learning disability and epilepsy. They compared this with the 33\% PASADD Checklist prevalence found by Roy et al (1997) in a community sample of 127 adults with learning disability identified from a learning disabilities register and through liaison with primary healthcare teams, and concluded that epilepsy in itself was not a risk factor for psychiatric disorder. However, normative data for the PAS-ADD Checklist used with adults with learning disability have been published since then (Taylor et al, 2004) showing a prevalence rate of $20.1 \%$, suggesting that there might indeed be an increased rate of psychiatric symptoms in people with epilepsy.

\section{Total prevalence and physical illness}

A relationship between physical and mental health has been demonstrated in the general population. In a community-based sample of people aged between 16 and 64 years with mild-to-moderate learning disability, Deb et al (2001a) found a statistically significant association of physical disability with psychiatric illness, but Moss et al (1993a) failed to demonstrate any such relationship in a community sample of people with severe learning disability aged over 50 years. This may have been due to the difficulties in identifying psychiatric disorders in people 
Box 2 Total prevalence of mental health problems in adults with learning disability

- The rate is considerably higher than that found in the general population

- It is probably between 30 and $50 \%$

- Its relationship with level of disability, gender, age, epilepsy and physical illness has not been fully ascertained

with severe learning disability and the confounding effect of increased physical morbidity with more severe levels of learning disability. Cooper (1997) found a relationship between physical ill-health and dementia in older adults with learning disability, but not between mental and physical ill-health.

The existing information on the total prevalence of mental health problems in adults with learning disability is summarised in Box 2.

\section{Life events and abuse}

Although conclusive evidence on the relationship between mental ill-health and factors such as level of learning disability and physical illness is still lacking, there has been some progress in our understanding of life events as possible aetiological factors. A recent study by Hastings et al (2004) measured the rate of life events in the preceding year for a large sample of adults with learning disability in the north of England. They found that $46.3 \%$ had experienced one or more life events, a rate similar to that in the general population. They also found that the presence of one or more life events in the preceding year was significantly associated with scoring above threshold on the affective/neurotic sub-scale of the PAS-ADD Checklist. There are some limitations to this study, but it does suggest that life events are associated with affective and neurotic symptoms in adults with learning disability.

When comparing a group of 50 people with learning disability who had been bereaved with a matched control group, Hollins \& Esterhuyzen (1997) found a higher rate of depression, anxiety and adjustment disorders in the bereaved group. However, that group had also experienced more life events, which may have affected this result.

A systematic review of the literature on the clinical effects of sexual abuse in people with learning disability (Sequeira \& Hollins, 2003) found several studies suggesting that a range of psychopathology, including traumatic stress reactions, depression, anxiety and behavioural problems (e.g. aggression, self-injury and sexual behaviour) may follow sexual abuse. However, because of methodological limitations the results are not conclusive.
From these studies it seems likely that adults with learning disability experience at least as many life events and are at least as vulnerable to these as are adults without learning disability and that affective and neurotic disorders, and probably other types of mental ill-health, are associated. Further work evaluating the role of life events in the mental health of this population needs to be performed.

\section{Specific disorders}

The estimated prevalence rates for specific mental health problems taken from the population-based study by Cooper (1997), described earlier in this article, are listed in Table 1 . The rates have been rounded up to the nearest $0.5 \%$. Attention-deficit hyperactivity disorder is missing from the table as there have been no population-based studies measuring this in adults with learning disability. Historically, the diagnosis of attention-deficit disorder in this population has been overlooked, but there is a growing interest in it and further epidemiological information is likely to be available in the future. The rates for some of the specific disorders are considerably higher than those in the general population and there are many possible biopsychosocial and developmental reasons for this.

The following sections discuss current knowledge about the epidemiology of particular disorders.

\section{Psychosis}

Most of the research literature on psychotic disorders focuses on people with mild learning disability. There is very little on psychosis in people with moderate, severe or profound disability. This is mainly because of the complexity of diagnosing these disorders in people with more severe levels of learning disability. First-rank symptoms are conceptually too difficult for most learning-disabled people to express and negative symptoms and thought disorder may be

Table 1 Estimated prevalence rates from populationbased studies of adults with learning disability

$\begin{array}{lr}\text { Disorder } & \text { Rate } \\ \text { Schizophrenia } & 3 \% \\ \text { Bipolar affective disorder } & 1.5 \% \\ \text { Depression } & 4 \% \\ \text { Generalised anxiety disorder } & 6 \% \\ \text { Specific phobia } & 6 \% \\ \text { Agoraphobia } & 1.5 \% \\ \text { Obsessive-compulsive disorder } & 2.5 \% \\ \text { Dementia at age 65 years and over } & 20 \% \\ \text { Autism } & 7 \% \\ \text { Severe problem behaviour } & 10-15 \%\end{array}$


inappropriately counted as symptoms of psychosis when they are in fact due to institutionalisation or are developmentally appropriate. Nevertheless, it is recognised that there is an increased prevalence of psychotic disorder in people with learning disability compared with the general population and this is one of the most consistent findings in the literature.

Several studies have reported the prevalence of schizophrenia in learning disability as 3\% (and this is probably an underestimate) compared with $1 \%$ for the general population. The explanation for this increased prevalence of psychosis in people with learning disability and the direction of the relationship is much less apparent. The concept of pfropfschizophrenie was revisited by Doody et al (1998) in a between-group study comparing people with mild learning disability and a history of schizophrenia and two control groups: one of people with schizophrenia but no learning disability and the other of people with mild learning disability but no history of psychosis. They found that individuals with schizophrenia and learning disability had a tendency to belong to families with a history of both disorders, showed a high rate of chromosomal variants in karyotypic testing and were more likely to have negative symptoms, epilepsy, neurological soft signs and episodic memory deficits than individuals with schizophrenia and no learning disability. The authors concluded that a severe form of schizophrenia may occur where schizophrenia and learning disability arise together from a common genetic aetiology.

Other genetic associations with psychosis that have been described include Prader-Willi syndrome and velocardiofacial syndrome. There are three genetic subtypes of Prader-Willi syndrome (deletion, maternal disomy and imprinting defect) and, interestingly, the association with affective psychosis is now known to relate principally to the genetic subtype of maternal disomy. One study found a 100\% prevalence of psychotic symptoms by age 28 years in a sample of people with this genetic subtype of the syndrome (Boer et al, 2002).

There have been no other recent epidemiological studies that have examined psychotic disorders in adults with learning disability.

\section{Affective disorders}

Affective disorder in people with learning disability has attracted a high level of research interest, but the unresolved difficulties encountered in its assessment and diagnosis have hampered progress in determining prevalence rates and associated factors. Most authors agree that standard diagnostic criteria are appropriate for diagnosing depression in people with mild learning disability, but there is some disagreement as to whether modifications are required for people with moderate-to-profound learning disability. Furthermore, the debate as to whether behavioural disorder is an atypical symptom of depression is yet to be resolved. However, from the studies that have been carried out it seems that the point prevalence of depression in people with learning disability is around $3-4 \%$. This is considerably higher than the $1.7 \%$ found in the general population (Meltzer et al, 1995).

Risk factors for depression in the general population include stress, life events, low socio-economic status, lack of social support, old age and female gender. These factors are shared by people with learning disability, who may be further disadvantaged because of their limited coping skills, experiences of discrimination, rejection, ridicule, stigma and abuse. However, whether they are also risk factors for depression in learning disability has not been adequately studied, although some results have been published. For example, Meins (1993) found that both men and women with little support tended to have more symptoms of depression than well-supported individuals. Lunsky (2003) found that learning-disabled individuals with higher selfreported depression scores were lonelier and had higher stress levels than individuals with lower scores. None of the population-based studies have demonstrated a relationship between affective disorder and gender in learning disability, but this may be because of a lack of power due to the small sample sizes. However, a study by Hastings et al (2004) of a population-based sample of over 1000 adults with learning disability demonstrated a significantly increased rate of affective and neurotic symptoms, as measured by the PAS-ADD Checklist, in women than in men.

These results do suggest that some of the risk factors for depression in people with learning disability might be the same as those for the general population. There is also the possibility that additional and as yet unknown factors are involved in the aetiology of depression in people with learning disability. An interesting study by Richards et al (2001) demonstrated a fourfold increased risk of affective disorders for people with mild learning disability even after controlling for social and material disadvantages and medical conditions. A higher prevalence of depression in adults with Down's syndrome than in adults with other causes of learning disability has been suggested by some researchers (although not found by others) and a relative serotonin deficiency has been proposed as the reason for this increased vulnerability (Collacott, 1999). Clearly, further research in this area is required to confirm this and establish whether there are any treatment implications. 


\section{Anxiety disorder and obsessive-compulsive disorder}

The identification of anxiety and stress disorders in people with learning disability is particularly difficult and they are frequently missed by carers and clinicians, primarily owing to communication problems. In severe and profound learning disability only behavioural symptoms can be assessed, and as a result many anxiety disorders are misdiagnosed as problem behaviours. Classification is also difficult because anxiety can be a perfectly normal and appropriate reaction but it also occurs in a number of very different psychiatric conditions. As a result of these difficulties, much of the epidemiology of anxiety disorders in adults with learning disability is still unknown and studies have tended to describe the occurrence of anxiety symptoms rather than specific disorders. A few studies have examined the types of fear of adults with learning disability and they seem similar to those reported by children of equivalent developmental age (except that adults with learning disability report more fears of doctors). However, the usual gender difference (female gender is associated with greater reporting of fears) has not been found. When a group of 30 adults with learning disability were compared with a similar group without learning disability, the learning disability group reported more fears (Pickersgill et al, 1994). The authors suggested that overprotection and poor linguistic skills, leading to greater difficulties in discussing or dismissing fears and resulting in overgeneralisation, were responsible for this.

Other suggested aetiological factors for anxiety disorders in people with learning disability include the effects of deinstitutionalisation and certain behavioural phenotypes. Fragile- $X$ syndrome is associated with social anxiety, and phenylketonuria and Williams syndrome with generalised anxiety.

Obsessional thoughts are hard for people with learning disability to describe and, although compulsions are more readily observed, it can be difficult to distinguish them from stereotypies, tics and autism. Despite these diagnostic problems, Cooper (1997) found a rate of $2.5 \%$ for obsessive-compulsive disorder in learning disability, which is higher than in the general population. The most common compulsions in the general population are hand-washing, checking and cleaning, but in adults with learning disability, ordering is the most common, with handwashing, cleaning and checking rarely seen.

\section{Behavioural disorders}

Some researchers believe that problem behaviours are symptoms of underlying psychiatric disorder, whereas others think that they are purely learned.
All agree that problem behaviours are common in learning disability (although exactly how common very much depends on the definition of problem behaviour used and the sample studied). Qureshi \& Alborz (1992) focused on highly disruptive, seriously challenging behaviour and found a prevalence rate of $5.7 \%$. In a similar study, Emerson et al (2001) found that $10-15 \%$ of people known to local education, social and healthcare services showed challenging behaviour. At the other end of the spectrum, Deb et al (2001b) found a rate of $60.4 \%$ for any behavioural disorder of any frequency or severity.

In people with learning disability, the most common forms of problem behaviour identified by the various studies are demanding behaviour, verbal aggression, physical aggression, destructiveness and self-injury. The majority of those with problem behaviour have more than one type, with some having as many as five different problem behaviours. Most studies have found a higher prevalence of problem behaviours in males, although Deb et al (2001b) found a significantly higher prevalence of severe problem behaviours in females. The general trend is towards a greater prevalence of problem behaviour with increasing severity of learning disability, although people with profound learning disability tend to exhibit less outwardly directed behaviour because of their restricted mobility.

The overall prevalence of problem behaviour in learning disability increases with age during childhood, reaches a peak in the age range 15-34 years and then declines. Thompson \& Reid (2002) found that a high number of behavioural symptoms persisted over a 26-year period in a cohort of 53 adults but the severity decreased. Moss et al (2000) found that increasing severity of challenging behaviour was associated with increased prevalence of psychiatric symptoms as measured by the PASADD Checklist, with depression showing the most marked association. These authors also found selfinjury to be associated with anxiety symptoms, a relationship suspected by other researchers. A study by Collacot et al (1998) on a population-based sample of 2277 adults found a prevalence of $1.7 \%$ for severe self-injury. No gender difference was apparent but self-injurious behaviour was associated with younger age, lower IQ, autistic symptoms, hearing impairment and immobility.

Another aetiological factor in problem behaviour is genetic disorder. Prader-Willi, Lesch-Nyhan, Aicardi, Rubenstein-Taybi and Smith-Magenis syndromes are all associated with self-injury. Angelman syndrome is associated with impulsivity and overactivity, Sotos syndrome with aggression and Cri du Chat syndrome with irritability. Physical health problems such as toothache and any febrile illness are also important precipitants of problem 
Box 3 Problem behaviours in learning disability

- Have a prevalence rate of $10-15 \%$

- Are more common in males

- Are most prevalent in the age range 15-34 years

- Have greater prevalence with increasing severity of learning disability

- More than one type of problem behaviour is usually found

- Tend to be longstanding

behaviour. Although some studies have found an increased rate of problem behaviour in people with epilepsy, others have found the opposite, and it remains unknown whether epilepsy plays a role in the aetiology of problem behaviour. The epidemiology of problem behaviours in adults with learning disability is summarised in Box 3 .

\section{Conclusions}

Much of the epidemiology of mental health problems in adults with learning disability is still unknown. Most of the research in this area has been carried out on biased samples using inadequate methods of identification, and consequently few results are generalisable. Recent developments include the publication of diagnostic criteria specifically for use in this population (DC-LD) and the development of tools for the identification and diagnosis of mental health problems with reasonable reliability and validity (PAS-ADD).

There have been only two population-based prevalence studies in the past decade. No study to date has measured the incidence of mental health problems in adults with learning disability. The total prevalence of mental health problems (including problem behaviour) in adults with learning disability is higher than in the general population, with a rate that lies somewhere between 30 and $50 \%$. Exactly how much higher it is and why remain unclear. Similarly, it is unclear what effect age, gender, physical illness, epilepsy or level of learning disability have on mental health problems. Information on the factors associated with specific mental health problems in people with learning disability is emerging, but much is still unknown.

\section{References}

Aman, M. G., Singh, N. N., Stewart, A. W., et al (1985) The Aberrant Behavior Checklist: a behavioral rating scale for the assessment of treatment effects. American Journal of Mental Deficiency, 89, 485-491.
American Psychiatric Association (1994) Diagnostic and Statistical Manual of Mental Disorders (4th edn) (DSM-IV). Washington, DC: APA.

Boer, H., Holland, A., Whittington, J. et al (2002) Psychotic illness in people with Prader-Willi syndrome due to chromosome 15 maternal uniparental disomy. Lancet, 359, 135-136.

Collacott, R. A. (1999) People with Down syndrome and mental health needs. In Psychiatric and Behavioural Disorders in Developmental Disabilities and Mental Retardation (ed. N. Barnes), p. 203. Cambridge: Cambridge University Press.

Collacott, R. A., Cooper, S. A, Branford, D., et al (1998) Epidemiology of self-injurious behaviour in adults with learning disabilities. British Journal of Psychiatry, 173, 428432.

Cooper, S. A. (1997) Epidemiology of psychiatric disorders in elderly compared with younger adults with learning disabilities. British Journal of Psychiatry, 170, 375-380.

Cooper, S. A. \& Bailey, N. M. (2001) Psychiatric disorders amongst adults with learning disabilities: prevalence and relationship to ability level. Irish Journal of Psychological Medicine, 18, 45-53.

Corbett, J. A. (1979) Psychiatric morbidity and mental retardation. In Psychiatric Illness and Mental Handicap (eds F. E. James \& R. P. Snaith), pp. 11-25. London: Gaskell Press. Costello, H., Moss, S., Prosser, H., et al (1997) Reliability of the ICD-10 version of the Psychiatric Assessment Schedule for Adults with Developmental Disability (PAS-ADD). Social Psychiatry and Psychiatric Epidemiology, 32, 339-343.

Day, K. (1985) Psychiatric disorder in the middle-aged and elderly mentally handicapped. British Journal of Psychiatry, 147, 660-667.

Deb, S. \& Joyce, J. (1998) Psychiatric illness and behavioural problems in adults with learning disability and epilepsy. Behavioural Neurology, 11, 125-129.

Deb, S., Thomas, M. \& Bright, C. (2001a) Mental disorder in adults with intellectual disability. 1: Prevalence of functional psychiatric illness among a community-based population aged between 16 and 64 years. Journal of Intellectual Disability Research, 45, 495-505.

Deb, S., Thomas, M. \& Bright, C. (2001b) Mental disorder in adults with intellectual disability. 2: The rate of behaviour disorders among a community-based population aged between 16 and 64 years. Journal of Intellectual Disability Research, 45, 506-514.

Doody, G. A., Johnstone, E. C., Sanderson, T. L., et al (1998) 'Pfropfschizophrenie' revisited. Schizophrenia in people with mild learning disability. British Journal of Psychiatry, 173, 145153.

Emerson, E., Kiernan, C., Alborz, A., et al (2001) The prevalence of challenging behaviors: a total population study. Research in Developmental Disabilities, 22, 77-93.

Espie, C. A., Watkins, J., Curtice, L., et al (2003) Psychopathology in people with epilepsy and intellectual disability: an investigation of potential explanatory variables. Journal of Neurology, Neurosurgery and Psychiatry, 74, 1485-1492.

Feinstein, C., Kaminer, Y., Barrett, R. P., et al (1988) The assessment of mood and affect in developmentally disabled children and adolescents: the emotional disorders rating scale. Research in Developmental Disabilities, 9, 109-121.

Hastings, R. P., Hatton, C., Taylor, J. L., et al (2004) Life events and psychiatric symptoms in adults with intellectual disabilities. Journal of Intellectual Disability Research, 48, 4246

Hollins, S. \& Esterhuyzen, A. (1997) Bereavement and grief in adults with learning disabilities. British Journal of Psychiatry, 170, 497-501.

Lund, J. (1985a) Epilepsy and psychiatric disorder in the mentally retarded adult. Acta Psychiatrica Scandinavica, 72, $557-562$.

Lund, J. (1985b) The prevalence of psychiatric morbidity in mentally retarded adults. Acta Psychiatrica Scandinavica, 72, 563-570.

Lunsky, Y. (2003) Depressive symptoms in intellectual disability: does gender play a role? Journal of Intellectual Disability Research, 47, 6-27. 
Matson, J. K., Kazdin, A. E. \& Senatone, V. (1984) Psychometric properties of the Psychopathology Instrument for Mentally Retarded Adults. Applied Research in Mental Retardation, 5, 81-89.

Meins, W. (1993) Prevalence and risk factors for depressive disorders in adults with intellectual disability. Australian and New Zealand Journal of Developmental Disabilities, 18, 147156.

Meltzer, H., Gill, B., Petticrew M., et al (1995) The Prevalence of Psychiatric Morbidity among Adults Living in Private Households. OPCS Surveys of Psychiatric Morbidity in Great Britain, Report 1. London: HMSO.

Moss, S., Goldberg, D., Patel, P., et al (1993a) Physical morbidity in older people with moderate, severe and profound mental handicap, and its relation to psychiatric morbidity. Social Psychiatry and Psychiatric Epidemiology, 28, 32-39.

Moss, S., Patel, P., Prosser, H., et al (1993b) Psychiatric morbidity in older people with moderate and severe learning disability. I: Development and reliability of the patient interview (PASADD). British Journal of Psychiatry, 163, 471-480.

Moss, S., Ibbotson, B., Prosser, H., et al (1997) Validity of the PAS-ADD for detecting psychiatric symptoms in adults with learning disability (mental retardation). Social Psychiatry and Psychiatric Epidemiology, 32, 344-354.

Moss, S., Prosser, H., Costello, H., et al (1998) Reliability and validity of the PAS-ADD Checklist for detecting psychiatric disorders in adults with intellectual disability. Journal of Intellectual Disability Research, 42, 173-183.

Moss, S., Emerson, E., Kiernan, C., et al (2000) Psychiatric symptoms in adults with learning disability and challenging behaviour. British Journal of Psychiatry, 177, 452-456.

Patel, P., Goldberg, D. \& Moss, S. (1993) Psychiatric morbidity in older people with moderate and severe learning disability. II: The prevalence study. British Journal of Psychiatry, 163, 481-491.

Pickersgill, M. J., Valentine, J. D., May, R., et al (1994) Fears in mental retardation. Part 1 . Types of fears reported by men and women with and without mental retardation. Advances in Behaviour Research Therapy, 16, 277-296.

Prosser, H., Moss, S., Costello, H., et al (1998) Reliability and validity of the Mini PAS-ADD for assessing psychiatric disorders in adults with intellectual disability. Journal of Intellectual Disability Research, 42, 264-272.

Qureshi, H. \& Alborz, A. (1992) Epidemiology of challenging behaviour. Mental Handicap Research, 5, 130-145.

Reiss, S. (1988) The Reiss Screen for Maladaptive Behavior Test Manual. Worthington, OH: IDS Publishing.

Reiss, S. (1990) Prevalence of dual diagnosis in community based day programs in the Chicago metropolitan area. American Journal on Mental Retardation, 94, 578-585.

Richards, M., Maughan, B., Hardy, R., et al (2001) Long-term affective disorder in people with mild intellectual disability. British Journal of Psychiatry, 179, 523-527.

Roy, A., Martin, D. M. \& Wells, M. B. (1997) Health gain through screening - mental health: developing primary health care services for people with an intellectual disability. Journal of Intellectual and Developmental Disability, 22, 227-239.

Royal College of Psychiatrists (2001) DC-LD [Diagnostic Criteria for Psychiatric Disorders for use with Adults with Learning Disabilities/Mental Retardation] (Occasional Paper OP48) London: Gaskell.

Sequeira, H. \& Hollins, S. (2003) Clinical effects of sexual abuse on people with learning disability: critical literature review. British Journal of Psychiatry, 182, 13-19.

Taylor, J. L., Hatton, C., Dixon, L., et al (2004) Screening for psychiatric symptoms: PAS-ADD Checklist norms for adults with intellectual disabilities. Journal of Intellectual Disability Research, 48, 37-41.

Thompson, C. L. \& Reid, A. (2002) Behavioural symptoms among people with severe and profound intellectual disabilities: a 26-year follow-up study. British Journal of Psychiatry, 181, 67-71.

World Health Organization (WHO) (1992) The Tenth Revision of the International Classification of Diseases and Related Health Problems (ICD-10). Geneva: WHO.

\section{MCQs}

1 Methodological factors that affect results of prevalence studies of mental health problems in people with learning disability include:

a the definition of learning disability used

$b$ the definition of mental health used

c the diagnostic criteria used

d the sample selected

e the method used for the identification and assessment of cases.

2 The total prevalence rate (including problem behaviour) of mental health problems in adults with learning disability is probably between:

a 5 and $25 \%$

b 50 and $80 \%$

c 30 and $50 \%$

d 5 and $10 \%$

e 10 and $60 \%$.

3 Life events in adults with learning disability:

a occur at a rate similar to that in the general population

$\mathrm{b}$ are unlikely to have any emotional impact on them

c have been associated with anxiety and depressive symptoms

d have been associated with psychotic disorders

e require further research to determine their role in the aetiology of mental health problems.

4 The most common forms of problem behaviour in learning disability include:

a self-injury

b stripping

c verbal aggression

d wandering

e physical aggression.

5 The most common compulsion found in people with learning disability is:

a hand-washing

b checking

c ordering

d cleanliness

e touching.

\section{MCQ answers}

$\begin{array}{llllllllll}\text { 1 } & & \text { 2 } & & 3 & & 4 & & 5 & \\ \text { a } & \text { T } & \text { a } & \text { F } & \text { a } & \text { T } & \text { a } & \text { T } & \text { a F } \\ \text { b } & \text { T } & \text { b F } & \text { b F } & \text { b F } & \text { b F } \\ \text { c } & \text { T } & \text { c } & \text { T } & \text { c } & \text { T } & \text { c } & \text { T } & \text { c T } \\ \text { d T } & \text { d F } & \text { d F } & \text { d F } & \text { d F } \\ \text { e T } & \text { e F } & \text { e T } & \text { e T } & \text { e F }\end{array}$

